\title{
Performance Evaluation of Heart Rate Turbulence Detection Using an Extended IPFM Model
}

\author{
K Solem ${ }^{1}$, P Laguna $^{2}$, JP Martínez ${ }^{2}$, L Sörnmo ${ }^{1}$ \\ ${ }^{1}$ Signal Processing Group, Dept of Electrical \& Info Tech, Lund University, Lund, Sweden \\ ${ }^{2}$ Aragon Inst for Engineering Research, CIBER-BBN, Zaragoza University, Zaragoza, Spain
}

\begin{abstract}
In a previous study, we presented a new approach to characterize heart rate turbulence (HRT). An HRT detector was developed which involved a HRT test statistic $T(\mathbf{x})$, being based on Karhunen-Loève (KL) basis functions. The purpose of the present study is to evaluate the performance of $T(\mathbf{x})$ on simulated data and compare to that of turbulence onset (TO) and turbulence slope (TS). Three different simulations were performed for the purpose of studying the influence of signal-to-noise ratio (SNR), $Q R S$ jitter, and ECG sampling rate. The results show that $T(\mathbf{x})$ performs strikingly better in all simulations.
\end{abstract}

\section{Introduction}

The short-term fluctuation in heart rate which may follow a ventricular ectopic beat (VEB) is referred to as HRT $[1,2]$. In normal subjects, the heart rate first increases and then decreases to baseline, immediately after a VEB. The increase in heart rate is hypothesized to be due to compensation of the sudden drop in blood pressure induced by the VEB and subsequently sensed by the baroreceptors [3]. Once blood pressure is restored, the heart rate returns to baseline in order to maintain the blood pressure. The subject's ability to recover from a local decrease in blood pressure is reflected by the strength of turbulence. The absence of HRT reflects autonomic dysfunction [3].

It has been demonstrated that HRT is a powerful predictor of mortality after acute myocardial infarction [1, 2], and offers considerable potential in other areas as well [3, 4]. Several parameters for HRT characterization have been presented of which TO and TS are the most commonly employed. The parameter TO is the relative change of RR intervals enclosing a VEB, defined by the relative difference of the averages of the 2 normal RR intervals before and after the VEB. Since TO measures the relative change in RR intervals, negative values of TO imply heart rate acceleration following the VEB, whereas positive values imply heart rate deceleration. The parameter TS is defined by the steepest slope observed over five consecutive RR intervals in the first $15 \mathrm{RR}$ intervals following the VEB. Prior to computation of TO and TS, an average RR interval tachogram is often determined from available VEBs.

While both TO and TS have proven to be clinically useful, they are heuristic in nature as they do not result from modeling of the data. In a previous study, we extended the well-known integral pulse frequency modulation (IPFM) model to account for ectopic beats and HRT [4]. Inspired by the extended model, a technique for HRT characterization was presented which involved a set of KL basis functions, expressing HRT as a function of time as opposed to existing HRT parameters which are functions of beat index. An HRT test statistic $T(\mathbf{x})$ was then developed based on the KL representation. The purpose of the present study is to evaluate the performance of $T(\mathbf{x})$ on simulated data and compare to that of TO and TS.

\section{Methods}

\subsection{Extended IPFM model}

The original IPFM model was introduced to generate a series of occurrence times for normal sinus beats with known rate variability, reflecting basic electrophysiological properties of the sinoatrial node $[5,6]$. The model input signal is the sum of a DC level, accounting for mean heart rate, and a zero mean modulating signal, $m(t)$, accounting for variability due to parasympathetic and sympathetic activity, see Fig. 1(a). The input signal is integrated until a threshold $T_{0}$ is reached, representing the mean interval length between successive events. An event is created at time $t_{k}$ (the $k^{\text {th }}$ beat following the initial event at $t_{0}=0$ ) as the output of the model, and the integrator is reset to 0 . As a result, the output signal of the IPFM model becomes an event series which represents beat occurrence times.

During sinus rhythm the electrophysiological influence of a supraventricular ectopic beat (SVEB) may be viewed as a reset of the charging potentials in the sinoatrial node. In order to incorporate such a property in the IPFM model, the integrator has to be reset at the occurrence time $t_{l}^{s}$ cor- 

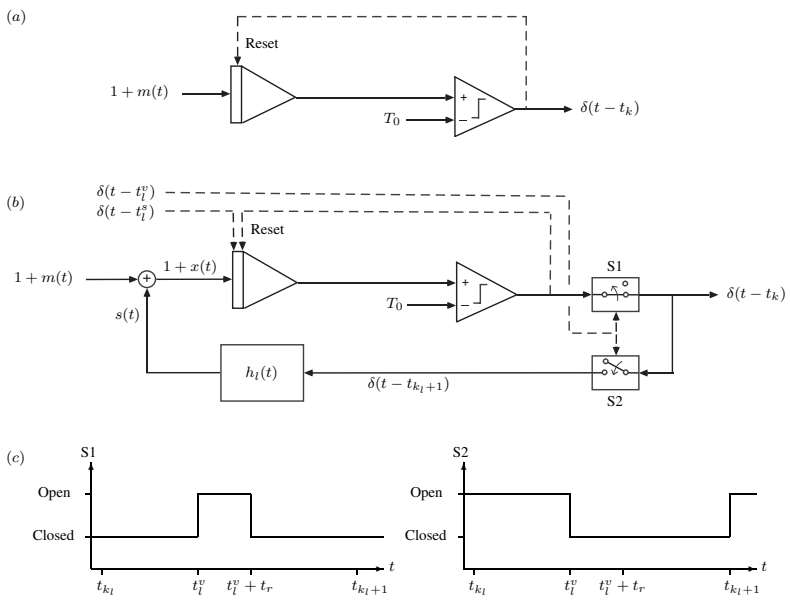

Figure 1. (a) Block diagram of the original IPFM model. (b) Block diagram of the extended IPFM model. (c) The switches, S1 and S2, in the extended IPFM model. The switches are drawn in the state of a normal sinus rhythm. Dashed lines describe control signals.

responding to the $l^{\text {th }}$ SVEB, see Fig. 1(b).

A normal heartbeat will induce an electrical wave propagating from the atria to the ventricles, whereas a VEB will induce a retrograde wave propagating from the ventricles to the atria. The electrical wave from a normal heartbeat will not arrive to the ventricles if a VEB is present, since the electrical wave will be interrupted by the retrograde wave induced by the VEB. In the extended model, this interruption is accounted for by the switch $\mathrm{S} 1$ which opens at $t_{l}^{v}$, corresponding to the occurrence time of the $l^{\text {th }}$ VEB, and which closes at $t_{l}^{v}+t_{r}$, where $t_{r}$ corresponds to the ventricular refractory period, see Figs. 1(b) and (c). These modifications account for SVEBs and VEBs when generating normal sinus beats without turbulence.

In order to account for the HRT phenomenon, additional feedback is introduced in the model. Physiologically, HRT is triggered at $t_{k_{l}+1}$ by the diastolic blood pressure drop induced by the VEB, where $t_{k_{l}+1}$ denotes the occurrence time of the first normal sinus beat that follows the $l^{\text {th }}$ VEB. The first normal sinus beat before the $l^{\text {th }}$ VEB is denoted $t_{k_{l}}$, so the time sequence is $\ldots, t_{k_{l}}, t_{l}^{v}, t_{k_{l}+1}, \ldots$. Heart rate turbulence is incorporated by feedback involving a linear system with causal impulse response $h_{l}(t)$, where $h_{l}(t)$ is the HRT associated with the $l^{\text {th }}$ VEB, see Fig. 1(b). The feedback branch models the baroreceptors so that HRT is triggered by an impulse fed to the linear system at $t_{k_{l}+1}$.

The HRT is modeled as a linear combination of basis functions,

$$
\mathbf{h}_{l}=\mathbf{B} \boldsymbol{\theta}_{l},
$$

where $\mathbf{h}_{l}$ is an $N \times 1$ vector with the discrete representation of $h_{l}(t)$, $\mathbf{B}$ is an $N \times p$ matrix with the basis functions in its columns, and $\boldsymbol{\theta}_{l}$ is a $p \times 1$ weight vector associated with the $l^{\text {th }}$ VEB. Thus, this extended IPFM model accounts for variations in both HRT dynamics and amplitude through $\mathbf{B}$ and $\boldsymbol{\theta}_{l}$. In this study, data-dependent basis functions are considered and, in particular, the KL basis functions since these are optimal for a given set of data. The KL basis functions are obtained as the eigenvalues and eigenvectors of the mean correlation matrix from subjects with HRT.

\subsection{HRT detection}

Our approach to detect and characterize HRT is based on the extended IPFM model and the linear model in (1). The detection procedure is formulated as one in which HRT is absent (hypothesis $\mathcal{H}_{0}$ ) or present (hypothesis $\mathcal{H}_{1}$ ),

$$
\begin{array}{ll}
\mathcal{H}_{0}: & \mathbf{x}=\mathbf{m} \\
\mathcal{H}_{1}: & \mathbf{x}=\mathbf{B} \boldsymbol{\theta}+\mathbf{m} .
\end{array}
$$

Here, $\mathbf{x}$ is an $N \times 1$ vector with the observed data, $\mathbf{m}$ is an $N \times 1$ vector with random white noise characterized by a Gaussian probability density function (PDF) $\mathcal{N}\left(\mathbf{0}, \sigma^{2} \mathbf{I}\right)$ with $\sigma^{2}$ as unknown parameter, $\mathbf{B}$ is a known $N \times p(p \leq$ $N$ ) orthogonal matrix, and $\boldsymbol{\theta}$ is a $p \times 1$ vector with unknown weights.

The resulting test statistic $T(\mathbf{x})$ of the generalized likelihood ratio test (GLRT) is used for HRT detection [4]. Thus, $\mathcal{H}_{1}$ is decided if

$$
T(\mathbf{x})=\frac{N-p}{p} \frac{\hat{\boldsymbol{\theta}}_{\mathcal{H}_{1}}^{T} \hat{\boldsymbol{\theta}}_{\mathcal{H}_{1}}}{\mathbf{x}^{T} \mathbf{x}-\hat{\boldsymbol{\theta}}_{\mathcal{H}_{1}}^{T} \hat{\boldsymbol{\theta}}_{\mathcal{H}_{1}}}>\gamma^{\prime},
$$

where $\hat{\boldsymbol{\theta}}_{\mathcal{H}_{1}}=\mathbf{B}^{T} \mathbf{x}$ is the maximum likelihood estimation of $\boldsymbol{\theta}$ assuming $\mathcal{H}_{1}$ is true, and $\gamma^{\prime}$ is a threshold determined by a given probability of false alarm $P_{F A}$.

\section{Simulation and performance measures}

The extended IPFM model is used to evaluate the performance of HRT detection. It is assumed that HRT has a known, fixed shape, obtained from the KL representation of the averaged HRT from 84 VEBs in 31 patients with myocardial ischemia selected from the European ST-T database [7]. The underlying heart rate variability (HRV), being modeled by $m(t)$, is obtained as the output of a $7^{\text {th }}$ order autoregressive (AR) model with white noise as input [7].

Three types of simulations were performed in order to evaluate HRT detector performance. The first type evaluated performance at different signal-to-noise-ratios (SNRs). The SNR associated with the $l^{\text {th }}$ VEB was calculated according to,

$$
\mathrm{SNR}_{l}=10 \log _{10}\left(\frac{\mathbf{h}_{l}^{T} \mathbf{h}_{l}}{\mathbf{m}_{l}^{T} \mathbf{m}_{l}}\right),
$$


where $\mathbf{m}_{l}$ is an $N \times 1$ vector with the discrete representation of $m(t)$ associated with the $l^{\text {th }}$ VEB. Different SNRs were obtained by changing the relation between the energy in $\mathbf{h}_{l}$ and $\mathbf{m}_{l}$ (weighting $\mathbf{h}_{l}$ with a suitable factor) before adding them to the input of the extended model. Thus, the noise power was fixed, whereas the signal energy was changed to produce different SNRs.

The second type of simulation evaluated the influence of QRS detection inaccuracies on HRT detection. Such inaccuracies were studied by adding zero mean Gaussian noise to the occurrence times of the normal sinus beats $t_{k}$ produced by the extended model.

The third type of simulation evaluated the influence of different sampling rates $F_{r}$ of the original ECG signal on HRT detection. This was studied by adding zero mean uniform noise, $\left[-\frac{1}{2 F_{r}}, \frac{1}{2 F_{r}}\right]$, to the occurrence times $t_{k}$.

Performance was measured by probability of detection $P_{D}$ and probability of false alarm $P_{F A}$, estimated by

$$
P_{D}=\frac{N\left(\mathcal{H}_{1} \mid \mathcal{H}_{1}\right)}{N\left(\mathcal{H}_{1} \mid \mathcal{H}_{1}\right)+N\left(\mathcal{H}_{0} \mid \mathcal{H}_{1}\right)},
$$

and

$$
P_{F A}=\frac{N\left(\mathcal{H}_{1} \mid \mathcal{H}_{0}\right)}{N\left(\mathcal{H}_{1} \mid \mathcal{H}_{0}\right)+N\left(\mathcal{H}_{0} \mid \mathcal{H}_{0}\right)},
$$

respectively, where $N\left(\mathcal{H}_{i} \mid \mathcal{H}_{j}\right)$ denotes the number of $\mathcal{H}_{i}$ decisions when $\mathcal{H}_{j}$ is true. Thus, $N\left(\mathcal{H}_{1} \mid \mathcal{H}_{1}\right)$ denotes when HRT is present and detected, $N\left(\mathcal{H}_{0} \mid \mathcal{H}_{1}\right)$ when HRT is present but missed (missed turbulence), $N\left(\mathcal{H}_{1} \mid \mathcal{H}_{0}\right)$ when HRT is absent but detected (false alarm), and $N\left(\mathcal{H}_{0} \mid \mathcal{H}_{0}\right)$ when HRT is absent and missed.

Different values of $P_{F A}$ were obtained by changing the detection threshold for the HRT parameter of interest. In the second and third type of simulation, detection performance is presented using the receiver operating characteristic (ROC) in which $P_{D}$ is displayed versus $P_{F A}$.

\section{Results}

The detection parameter $P_{D}$ is displayed as a function of the SNR for a fixed $P_{F A}=0.05$ in order to relate the performance of the different HRT detectors to averaging, see Fig. 2. Here, it is implied that different SNRs may be interpreted as different numbers of VEBs used for averaging. The horizontal differences between the curves in Fig. 2 can be interpreted as a power gain of $T(\mathbf{x})$ with respect to TO and TS. For $P_{D}=0.95$, there is approximately a $6 \mathrm{~dB}$ gain between $T(\mathbf{x})$ and $\mathrm{TO}$, and a $3 \mathrm{~dB}$ gain between $T(\mathbf{x})$ and TS. This means that $T(\mathbf{x})$ achieves the same performance as TO, using only one fourth of the number of VEBs for averaging, and the same performance as TS with only half the number of VEBs, assuming that HRT and noise in different VEBs are uncorrelated [6].

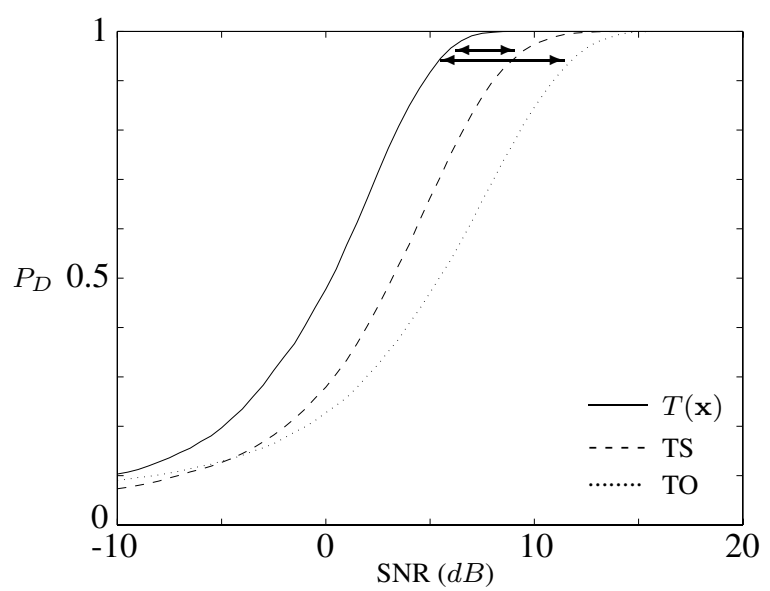

Figure 2. $P_{D}$ as a function of the SNR, for $P_{F A}=0.05$. The power gain is approximately $6 \mathrm{~dB}$ between $T(\mathbf{x})$ and TO, and approximately $3 \mathrm{~dB}$ between $T(\mathbf{x})$ and TS; for $P_{D}=0.95$.

The influence of QRS detection inaccuracies on HRT detection was studied for $T(\mathbf{x})$, TO, and TS, see Fig. 3. The detection performance of $T(\mathbf{x})$ is unaffected by QRS jitter with a standard deviation of $0.5 \mathrm{~ms}$, whereas TO and TS are slightly reduced, see Fig. 3. If the standard deviation is larger than $0.5 \mathrm{~ms}$, the performance of $T(\mathbf{x})$ is superior to both TO and TS, see Fig. 3.

The influence of the sampling rate $F_{r}$ of the original ECG signal on HRT detection was found to be negligible for all detectors when $F_{r}=1000 \mathrm{~Hz}$ was used, see Fig. 4. However, for lower rates, the performance of $T(\mathbf{x})$ was vastly superior to both TO and TS. For example, with a sampling rate of $F_{r}=250 \mathrm{~Hz}$ and a $P_{F A}=0.05$, the $P_{D}$ for $T(\mathbf{x})$, TS, and TO were $100 \%, 64 \%$, and $38 \%$, respectively, see Fig. 4.

\section{Conclusions}

The present paper demonstrates that the achieved performance of the model-based approach to HRT detection is superior to both TO and TS, to date being the two most common detection parameters. The simulation results show that the HRT test statistic $T(\mathbf{x})$ achieves the same performance as TO and TS at a considerably lower SNR. In order to attain the same performance as $T(\mathbf{x})$, TS needs at least twice the amount of VEBs, and TO at least four times. It is also shown that $T(\mathbf{x})$ performs much better in the presence of QRS jitter and at lower ECG sampling rates than do TO and TS. 

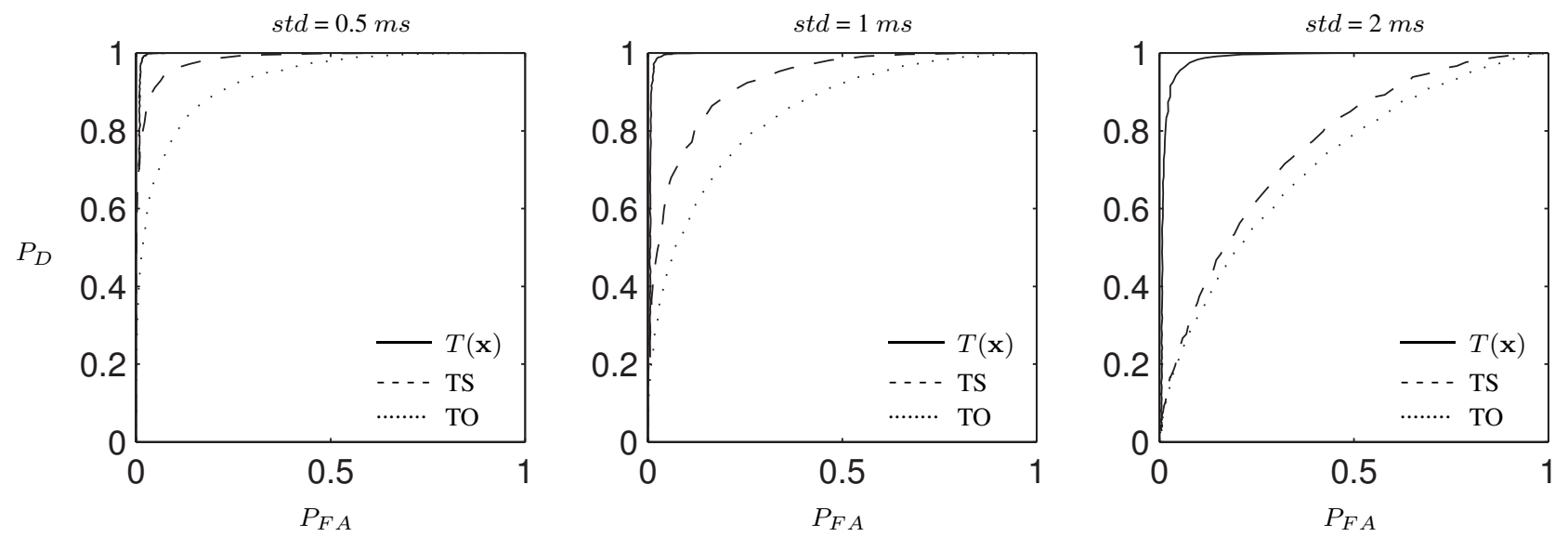

Figure 3. ROC curves illustrating the influence of QRS detection inaccuracies on HRT detection, studied by QRS jitter with a standard deviation (std) of $0.5 \mathrm{~ms}, 1 \mathrm{~ms}$, and $2 \mathrm{~ms}$. The SNR was equal to $10 \mathrm{~dB}$.
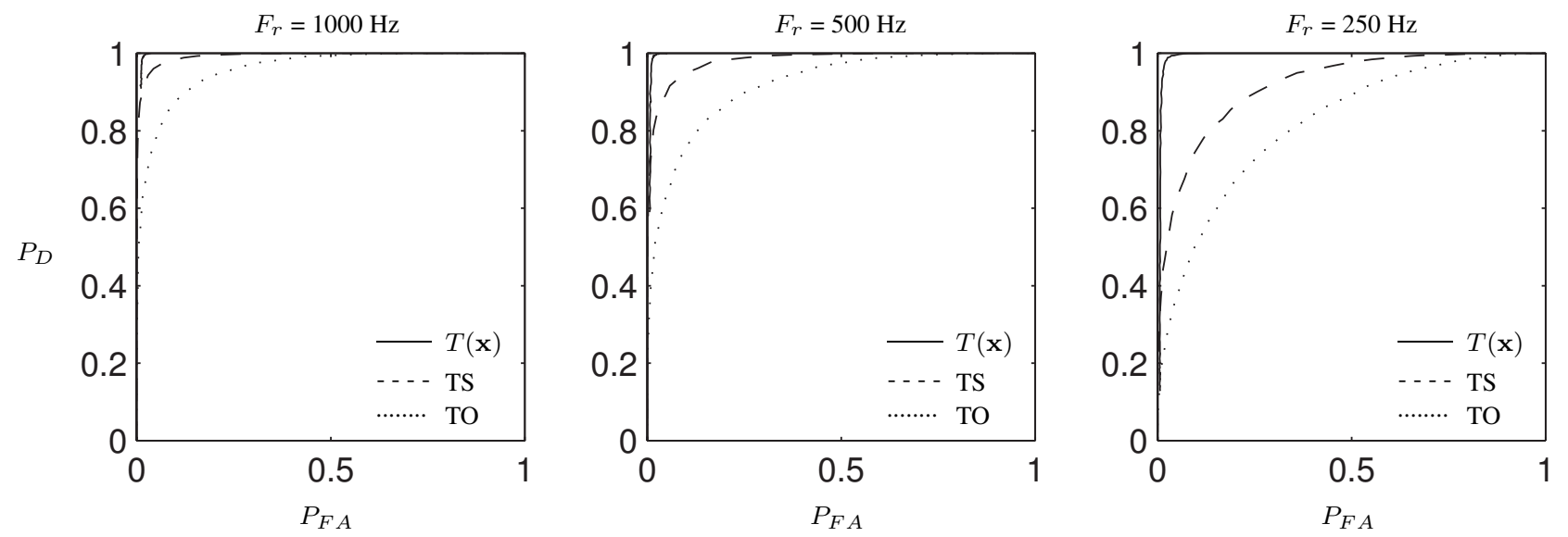

Figure 4. ROC curves illustrating the influence of sampling rate $F_{r}$ on HRT detection. The SNR was equal to $10 \mathrm{~dB}$.

\section{Acknowledgements}

This study was supported by a grant from Gambro AB.

\section{References}

[1] Schmidt G, Malik M, Barthel P, Schneider R, Ulm K, Rolnitzky L, Camm AJ, Bigger JT, Schömig A. Heart-rate turbulence after ventricular premature beats as a predictor of mortality after acute myocardial infarction. Lancet 1999; 353(9162):1390-1396.

[2] Schneider R, Barthel P, Watanabe M. Heart rate turbulence on Holter. In Malik M, Camm AJ (eds.), Dynamic electrocardiography. New York: Futura Publ., 2004; 190-193.

[3] Watanabe M, Schmidt G. Heart rate turbulence: A 5-year review. Heart Rhythm 2004;1:732-738.

[4] Solem K, Laguna P, Sörnmo L. Detection of heart rate tur- bulence using an extended IPFM model. In Proc. Computers in Cardiology, Valencia, Spain. 2006; 905-908.

[5] Hyndman BW, Mohn RK. A model of the cardiac pacemaker and its use in decoding the information content of cardiac intervals. Automedica 1975;1:239-252.

[6] Sörnmo L, Laguna P. Bioelectrical Signal Processing in Cardiac and Neurological Applications. Amsterdam: Elsevier (Academic Press), 2005.

[7] Mateo J, Laguna P. Improved heart rate variability signal analysis from the beat occurrence times according to the IPFM model. IEEE Trans Biomed Eng 2000;47:997-1009.

Address for correspondence:

Kristian Solem

Department of Electrical \& Info Tech, Lund University

Box 118, SE-221 00 Lund, Sweden

Kristian.Solem@eit.lth.se 